\title{
Invisible or Spontaneous Pneumothorax: An Unusual Presentation
}

\author{
Hassan Mumtaz ${ }^{1 *}$, Kaynat Mustafa ${ }^{2}$, Komal Safdar ${ }^{2}$, Fatima Meer $^{3}$, Tehreem Fatima ${ }^{4}$ and \\ Muhammad Ahsan Shafiq ${ }^{5}$ \\ ${ }^{1}$ House Surgeon, KRL Hospital Islamabad, Former House Physician, Holy Family Hospital Rawalpindi, Guys\& St Thomas Hospital \\ NHS Trust, UK
}

${ }^{2}$ House Physician, Dr Akbar Niazi Teaching Hospital Islamabad

${ }^{3}$ House Surgeon, Holy Family Hospital Rawalpindi

${ }^{4}$ House Surgeon, Holy Family Hospital Rawalpindi CIBNP, Fairfield USA

${ }^{5}$ Resident Physician Internal Medicine, Holy Family Hospital Rawalpindi CIBNP, Fairfield USA

*Corresponding author: Hassan Mumtaz, House Surgeon, KRL Hospital Islamabad, Former House Physician, Holy Family Hospital Rawalpindi, Guys \& St Thomas Hospital NHS Trust, UK

\section{ARTICLE INFO}

Received: 㗀 September 24, 2020

Published: 菛 October 02, 2020

Citation: Hassan Mumtaz, Kaynat Mustafa, Komal Safdar, Fatima Meer, Tehreem Fatima, Muhammad Ahsan Shafiq. Invisible or Spontaneous Pneumothorax: An Unusual Presentation. Biomed J Sci \& Tech Res 30(5)-2020. BJSTR. MS.ID.005022.

\begin{abstract}
Pneumothorax is a frequent, life-threatening complication, specifically in critically ill patients.In this report, we describe two unusual cases, in which one of them presented in the emergency department while the other came in the outdoor department. Case 1 was diagnosed as having Primary Spontaneous Pneumothorax, while in case 2 had a pneumothorax secondary to pulmonary edema. We discuss the clinical presentation, risk factors, and therapeutic options.Physicians should always have a low threshold for the diagnosis and regularly followup patients to prevent spontaneous pneumothorax
\end{abstract}

\section{Introduction}

Pneumothorax is a frequent, life-threatening complication, specifically in critically ill patients. Occult pneumothorax has been defined as pneumothoraces seen on CT scans or chest ultrasound but not on routine chest radiographs and may rapidly progress to tension pneumothorax. The phenomenon of occult pneumothorax is well described in the literature with an incidence of between 2 and $12 \%$ in medical and trauma patients [1]. Spontaneous Pneumothorax (SP) is a rare disorder that historically affects approximately $0.5-11.6 \%$ of patients [2]. Non-traumatic Pneumothorax is 30 times more common in men than in women, and the gender difference is considerably larger [3]. Primary SP (PSP) occurs in a previously healthy patient without underlying chronic lung disease or provoking factors, such as trauma, surgery, or diagnostic intervention, while secondary SP is associated with parenchymal lung disease, such as pulmonary fibrosis or emphysema [4].

In this report, we describe two unusual cases, in which one of them presented in the emergency department while the other came in the outdoor department. Case 1 was diagnosed as having Primary Spontaneous Pneumothorax, while in case 2 had a pneumothorax secondary to pulmonary edema. We discuss the clinical presentation, risk factors, and therapeutic options.

\section{Case No.1}

A 32-year-old male presented to OPD of Holy Family Hospital Rawalpindi with asymptomatic left sided Hemo-Pneumothorax which was diagnosed during routine occupational checkup. He had no complaints of shortness of breath or chest pain but there was 
a history of high-grade fever, which was on and off for 2 months, relieved by panadol. It is to be noted that there was history of similar iatrogenic pneumothorax a year ago which was treated with chest intubation for 6 days. Chest tube was removed after complete resolution of pneumothorax.On examination respiratory rate of 18 breaths per minute was noted. Palpation revealed bilateral equal chest expansion. On percussion a slightly dull note was noted on left lower lung and auscultation revealed a decreased air entry in left lower lung field. Chest X-ray showed left sided hydro pneumothorax with pleural effusion. Chest intubation was done, and patient was managed conservatively. However, he did not improve even after 10 days of chest intubation. He developed intermittent fever and became tachypnic. Therefore, thoracotomy and decortication was done. Per-op findings were Left sided fibro-thorax and thickened parietal and visceral pleura(Figure 1).

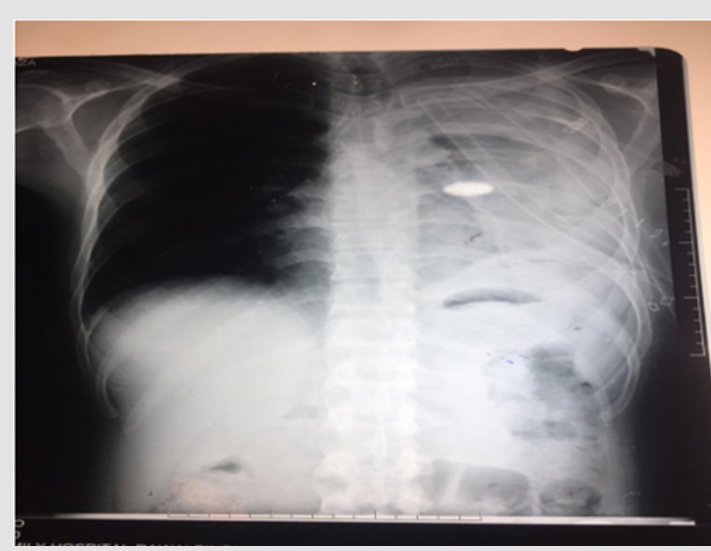

Figure 1: showing a shrunken left lung.

During surgery left lung was inflated and lung expansion was observed.Pleural tissue biopsy reported acute chronic inflammation with formation of granulation tissue.Unfortunately patient's post -op xray showed collapsed left lung. Ultrasound done on 6th post-op day indicated infected fluid collection in left pleural cavity representing left sided moderate empyma. No improvement was observed on conservative management. Rethoracotomy and pleural wash was done at 25th post-op day. Perop findings revelaed clots in left pleural cavity, which were removed and decortication was done. Pleural cavity was washed with $10 \mathrm{~L}$ normal saline. Chest tubes were placed anterior and posterior to lung. Thick piece of cortex around lung was sent for biospy which was consistent with empyma thoracis, however it was negative for granulomatous inflammation or malignancy. Post-op xray showed normally expanded left lung. The patient recovered well and was discharged.

\section{Case No. 2}

A 81 year old man presented into the emergency department of KRL Hospital Islamabad with shortness of breath and Acute Kidney Injury.According to patient's attendant he was in unusual state of health when he developed mild burning micturition along with low grade fever which was relieved by panadol. He had shortness of breath from $0_{2}$ years which progressed from MMRC grade 2 to grade 4. He also had an history of Hypertension, Ischemic Heart Disease having an Ejection Fraction 25\%, along with pulmonary edema.Initially patient was maintaining $\mathrm{O}_{2}$ stats at room air but then started on $\mathrm{O}_{2}$ therapy and later on shifted to nasal cannula. He was well oriented in time and space but then he became critically ill due to multi organ involvement. He was confused, tachypnic and not following any commands(Figure 2). His blood pressure was 168/98 $\mathrm{mm} \mathrm{Hg}$ where as blood sugar random measured was $140 \mathrm{mg} / \mathrm{dl} /$. ECG showed hyper acute t wave along with prolonged PR interval. Chest examination revealed bilateral creptsupto $2 / 3^{\text {rd }}$ of chest with mild wheeze. Ultrasound abdomen had a bilateral echogenic kidney, mild hydronephrosis along with mild intrahepatic cholestasis. Chest $\mathrm{x}$-ray revealed bilateral infiltrates reticular in base and periphery along with central consolidation giving an impression of Right Pneumothorax Table 1. Since his pneumothorax was less than $30 \%$, he was kept under observation and managed conservatively. His current line of treatment was meropenem, azithromycin, carvedilol, asprin, steroids along side with nebulization of Atem and Clenil.

Table 1: ECG showed hyper acute $t$ wave along with prolonged PR interval.

\begin{tabular}{|c|c|}
\hline TLC & 23.2 \\
\hline HB & 12.5 \\
\hline PLT & 67 \\
\hline CRP & 172.6 \\
\hline Total Bilirubin & 3.89 \\
\hline ALT & 123 \\
\hline ALP & 300 \\
\hline Urea & 127 \\
\hline Creatinine & 3.1 \\
\hline Na $^{+}$ & 146 \\
\hline $\mathrm{K}^{+}$ & 3.3 \\
\hline Ca $^{+}$ & 7.9 \\
\hline Albumin & 2.7 \\
\hline
\end{tabular}

Chest examination revealed bilateral creptsupto $2 / 3$ rd of chest with mild wheeze. Ultrasound abdomen had a bilateral echogenic kidney, mild hydronephrosis along with mild intrahepatic cholestasis

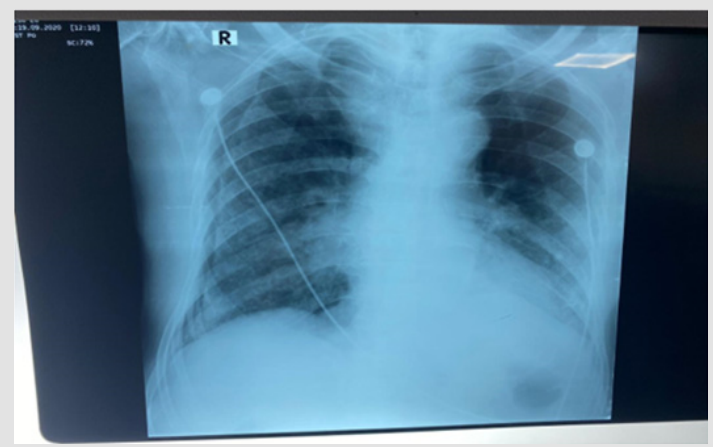

Figure 2: Showing Right Pneumothorax. 


\section{Discussion}

Spontaneous pneumothorax in a patient who had no underlying medical condition is called Bilateral primary spontaneous pneumothorax (BPSP). Even though the definite mechanism is still not clear, rupture of the blebs in the lung is the most commonly known cause of the BPSP [5].Among these patients, some would present as Bilateral Primary Spontaneous Pneumothorax, a rare presentation with limited data on incidence and prognosis. Some of the following characteristics may be seen in patients with BPSP:the patients tend to be younger, with low body mass index, the patients may have a previous history of spontaneous unilateral pneumothorax, and the size of pneumothorax is usually small [67]. In contrast, the patient in our case no 1, presented without any underlying disease.Approximately $58 \%$ and $68 \%$ of patients with Bilateral Secondary Spontaneous Pneumothorax (BSSP), as in our case no 2 had causative underlying lung disease, which can include pulmonary metastases, tuberculosis, Ehlers-Danlos syndrome, histiocytosis $\mathrm{X}$, and COPD $[5,8]$. Most recur within approximately 6-12 months after the first episode [3,4].

There are no specific guidelines for the management of Spontaneous Hemo Pneumothorax (SHP). The clinical features in SHP are dramatic and depend on the volume of blood loss and the amount of air leakage $[8,9]$. SHP can be life-threatening, and aggressive management is required. When SHP becomes lifethreatening because of substantial bleeding, the early insertion of two large bore chest drains to evacuate the accumulated blood and air is recommended to permit the re-expansion of the lung, as done in our case no 1 , which can result in hemostasis by compressing the bleeding vessels against the parietal pleura [9].Surgical strategies for the management of PSP include open thoracotomy as done in our case no.1 and pleurectomy or video-assisted thoracoscopic surgery (VATS) with pleurectomy and pleural abrasion. An open approach offers lower recurrence rates, while a less invasive approach with VATS has the advantages of less postoperative pain, improved pulmonary function, and a decreased length of hospital stay [10-11].

\section{ISSN: 2574-1241}

DOI: $10.26717 /$ BJSTR.2020.30.005022

Hassan Mumtaz. Biomed J Sci \& Tech Res

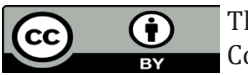

This work is licensed under Creative Commons Attribution 4.0 License

Submission Link: https://biomedres.us/submit-manuscript.php

\section{Conclusion}

Spontaneous pneumothorax may rapidly progress to a fatal tension pneumothorax if left untreated. Thus, physicians should always have a low threshold for this diagnosis and work-up for this condition. In patients who have recovered from pneumothorax should be regularly followed up to prevent primary spontaneous pneumothorax. Similarly, patients having underlying diseases should be advised to consult experts to prevent the development of secondary spontaneous pneumothorax.

\section{References}

1. Carrillo-Esper R, Sosa-García JO (2012) Hidden pneumothorax, a frequent but under-diagnosed pathology. Med Sur 19(2): 88-92.

2. Williams EW, Ramphal PS, Williams-Johnson J, Ford R, McDonald $\mathrm{AH}$, et al. (2005) Spontaneous haemo-pneumothorax: a rare but lifethreatening phenomenon. West Indian Med J 54(5): 346-347.

3. De Perrot M, Deleavel J, Robert J, et al. (2000) Spontaneous hemopneumothorax-results of conservative treatment. Swiss Surg 6(2): 62-64.

4. Boersma W, Stigt J, Smit H (2010) Treatment of haemothorax. Respir Med 104: 1583-1587.

5. TI Akcam, O Kavurmaci, A G Ergonul, et al. (2018) "Analysis of the patients with simultaneous bilateral spontaneous pneumothorax." The Clinical Respiratory Journal 12(3): 1207-1211.

6. S C Lee, YL Cheng, CW Huang, C Tzao, HH Hsu, et al. (2008) "Simultaneous bilateral primary spontaneous pneumothorax." Respirology 13 (1): 145148.

7. TW Huang, YL Cheng, C Tzao, et al. (2007) "Factors related to primary bilateral spontaneous pneumothorax." The Thoracic and Cardiovascular Surgeon 55(5): 310-312.

8. A Sayar, A Turna, M Metin, N Küçükyağci, O Solak, et al. (2004) "Simultaneous bilateral spontaneous pneumothorax report of 12 cases and review of the literature." Acta Chirurgica Belgica 104(5): 572-576.

9. Por NC, Fai CL, Hung CC (2003) Spontaneous massive haemopneumothorax: case reports. Eur J Emerg Med 10(1): 47-51.

10. Muraguchi T, Tsukioka K, Hirata S, et al. (1993) Spontaneous hemopneumothorax with aberrant vessels found to be the source of bleeding: report of two cases. Surg Today 23(12): 1119-1123.

11. Chen Y, Guo Z (2018) Unusual case of primary spontaneous hemopneumothoraxin ayoung man with atypical tension pneumothorax: a case report. J Med Case Reports12:188.

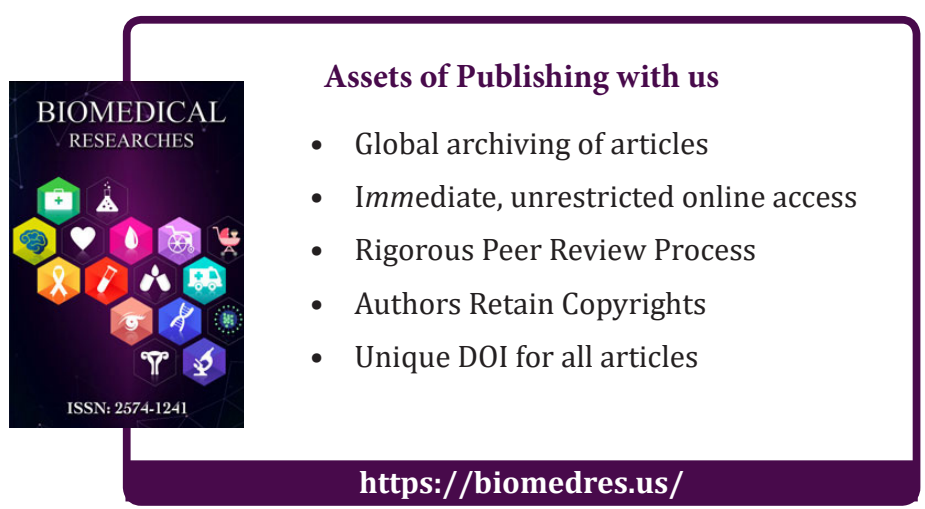

